\title{
Event-by-event analysis: Possible testing ground for the nuclear matter equation of state
}

\author{
G. Buchwald, G. Graebner, J. Theis, J. Maruhn, and W. Greiner \\ Institut für Theoretische Physik, Goethe Universität, Frankfurt am Main, Federal Republic of Germany \\ H. Stöcker \\ Department of Physics and Astronomy and National Superconducting Cyclotron Laboratory, \\ Michigan State University, East Lansing, Michigan 48824 \\ K. Frankel and M. Gyulassy \\ Nuclear Science Division, Lawrence Berkeley Laboratory, Berkeley, California 94720
}

(Received 20 April 1983)

\begin{abstract}
Intranuclear cascade calculations and fluid dynamical predictions of the kinetic energy flow are compared for collisions of ${ }^{40} \mathrm{Ca}+{ }^{40} \mathrm{Ca}$ and ${ }^{238} \mathrm{U}+{ }^{238} \mathrm{U}$. The aspect ratio, $R_{13}$, as obtained from the global analysis, is independent of the bombarding energy for the intranuclear cascade model. Fluid dynamics, on the other hand, predicts a dramatic increase of $R_{13}$ at medium energies $E_{\text {lab }} \lesssim 200$ $\mathrm{MeV} /$ nucleon. In fact, $R_{13}\left(E_{\mathrm{lab}}\right)$ directly reflects the incompressibility of the nuclear matter and can be used to extract the nuclear equation of state at high densities. Distortions of the flow tensor due to few nucleon scattering are analyzed. Possible procedures to remove this background from experimental data are discussed.
\end{abstract}

Experimental information on the properties of dense matter is being sought by studying the fragment emission pattern produced in high energy nuclear collisions. For example, the double differential cross sections of light fragments emitted from nearly central-i.e., high multiplicity selected-collisions of $\mathrm{Ne}(393 \mathrm{MeV} /$ nucleon $)$ $+\mathrm{U}$ have been measured ${ }^{1}$ and compared to the predictions of the different theoretical calculations. ${ }^{2}$ The measured angular distributions of the emitted protons in these central collisions exhibit strong forward suppression. In contrast to the data, the cascade calculations yield forward-peaked angular distributions, even if central collisions are selected. On the other hand, the fluid dynamical model, with final thermal breakup included, gives a reasonable description of the observed forward suppression.

Early emulsion experiments ${ }^{3}$ also showed sidewards peaking in the angular distribution of emitted $\alpha$ particles. Another indication for collective flow effects has recently been found in a two-particle correlation measurement ${ }^{4}$ : A fast sidewards moving proton evidently is preferentially emitted in coincidence with another proton moving in the same direction ("jetting phenomenon"2), rather than in the opposite direction as expected with knockout models. All these observations have remained inconclusive, however, due to alternate possible explanations such as Coulomb effects, depletion of phase space due to composite formation, and geometrical shadowing effects.

A recent idea on how the collective flow can be observed more directly is "global" momentum tensor analysis. This analysis can be done experimentally only with $4 \pi$ detector systems such as emulsion, streamer chamber, or the plastic ball. The basic idea is to measure event-by-event the momenta of all (charged) particles.
Once this information is available, one can transform all the physical quantities into the center-of-momentum frame and determine the direction of maximum momentum and energy flow by performing a principal axis transformation. The various concepts which have been proposed to analyze nuclear collisions are thrust, ${ }^{2,5,6}$ sphericity, ${ }^{6-8}$ and kinetic energy flow. ${ }^{7-9}$ The first two concepts have been adapted from high energy physics, but they have the disadvantage of being either nonanalytic (thrust) or of not properly taking into account the emission of composite particles (sphericity). The kinetic energy flow tensor, ${ }^{7-9}$

$$
F_{i j}=\sum \frac{p_{i}(v) p_{j}(v)}{2 m_{v}},
$$

where the sum runs over all fragments $v$ with mass and center of mass momentum $\overrightarrow{\mathrm{p}}(v)$, is a generalization of the sphericity concept. The factor $\frac{1}{2} m_{v}$ ensures that composite fragments contribute to the matter flow tensor with the correct weight relative to nucleons.

By comparing the results of the cascade and the hydrodynamic calculation, we want to determine the sensitivity of the global variables to the collision dynamics. In cascade calculations, $m_{v}=m_{N}$ and the $\overrightarrow{\mathrm{p}}(v)$ are the final momenta of all nucleons. In hydrodynamic calculations, the reaction volume is divided into cells $v$ characterized by a mean flow velocity, $\overrightarrow{\mathrm{p}}(v) / m_{N}$, a local temperature $T(v)$, and a local baryon number $N(v)$. When the baryon density in a cell falls below a freezeout value, $\rho_{f} \approx \rho_{0} / 2$, $\rho_{0}=0.15 \mathrm{fm}^{-3}$, it contributes an amount $\bar{p}_{i} \bar{p}_{j} / 2 \mathrm{~m}$ $+\delta_{i j} T / 2$ to the flow tensor. Thus, for hydrodynamics $F_{i j}$ is the sum of a collective flow energy $\bar{F}_{i j}$ and a thermal 


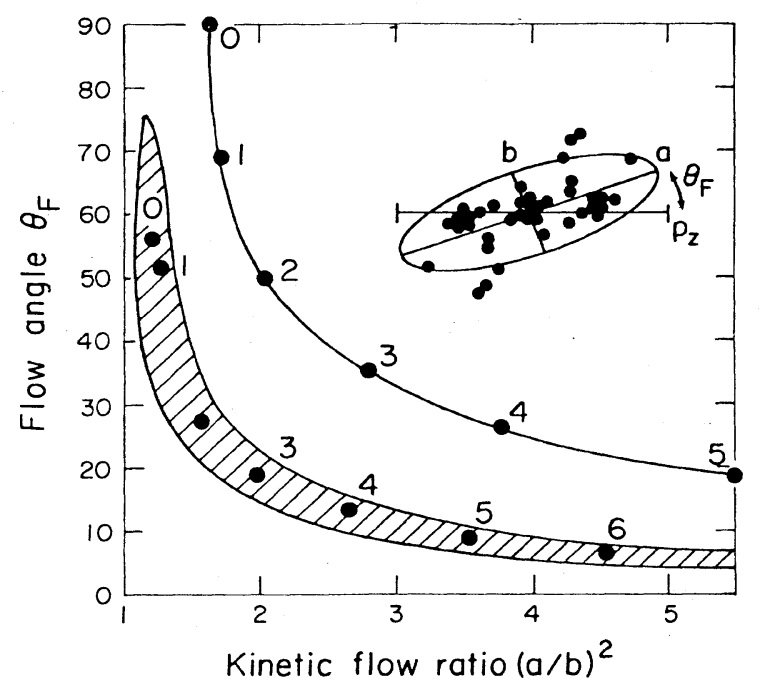

FIG. 1. The momentum flow analysis for $\mathrm{U}(400$ $\mathrm{MeV} /$ nucleon) $+\mathrm{U}$ is shown in the $R_{13}-\theta$ plane for the cascade model calculation (the shaded area indicating the width of the ridge) and the fluid dynamical calculation (solid line). The numbers indicate the impact parameter in fm's.

energy $\delta_{i j} E_{T} / 3$,

$$
F_{i j}=\sum_{v} \frac{\bar{p}_{i}(v) \bar{p}_{j}(v)}{2 m_{N}}+\delta_{i j} \frac{1}{2} T(v) \equiv \bar{F}_{i j}+\delta_{i j} E_{T} / 3 .
$$

Observe that the eigenvalues of $F$ are then $\lambda_{n}=\bar{\lambda}_{n}+E_{T} / 3$, where $\bar{\lambda}_{n}$ are the eigenvalues of $\bar{F}$. However, the eigenvectors, $\widehat{e}_{n}$, are identical to those of $\bar{F}$. Thus, while the aspect ratio, $R_{13}=\lambda_{1} / \lambda_{3}$, where $\lambda_{1}>\lambda_{2}>\lambda_{3}$, is brought closer to unity by thermal smearing, the flow angle $\theta_{F}=\cos ^{-1}\left(\widehat{e}_{1} \cdot \hat{z}\right)$ is unaffected by temperature.

In Fig. 1 we plot the flow angle $\theta_{\text {flow }}^{\text {c.m. }}$, i.e., the angle of the largest principle axis of the flow tensor to the beam axis, versus the aspect ratio $R_{13}$ for the reaction U(400 $\mathrm{MeV} /$ nucleon) $+\mathrm{U}$. Note that $R_{13} \gg 1$ reflects events stretched in momentum space, while $R_{13}=1$ indicates a spherical momentum distribution. The ridge in the $\left(\theta_{\text {flow }}, R_{13}\right)$ plane resulting from the cascade calculations depends on the total mass of the systems. The cascade calculations ${ }^{6,7}$ show that substantial flow angles should only be expected for very heavy systems $A_{1}=A_{2}>100$. Furthermore, there are substantial finite number distortions ${ }^{10}$ of the flow characteristics for $A<100$. Also shown are the results of the fluid dynamical calculation for the same system. Larger deflection angles and aspect ratios $R_{13}$ indicate that the matter flux is apparently more strongly correlated for the hydrodynamical model. The impact parameter dependence of the flow angle, $\theta_{F}$, aspect ratio $R_{13}$, sphericity $S=\frac{3}{2}\left(\lambda_{1}+\lambda_{2}\right)$, and coplanarity (or flatness) $C=\frac{3}{2}\left(\lambda_{2}-\lambda_{1}\right)$ with $\lambda_{1}<\lambda_{2}<\lambda_{3}$ the principal values [normalized by $\left(\operatorname{Tr} F_{i j}\right)^{-1}$ ], is shown in Fig. 2 from the hydrodynamic model calculation for the system ${ }^{40} \mathrm{Ca}(400 \mathrm{MeV} /$ nucleon $)+{ }^{40} \mathrm{Ca}$. Observe the greater sensitivity of $\theta_{F}, R_{13}$ to impact parameter than that of $S$ and $D$. Also note that $R_{13}=1.7$ at $b=0$ is remarkably close

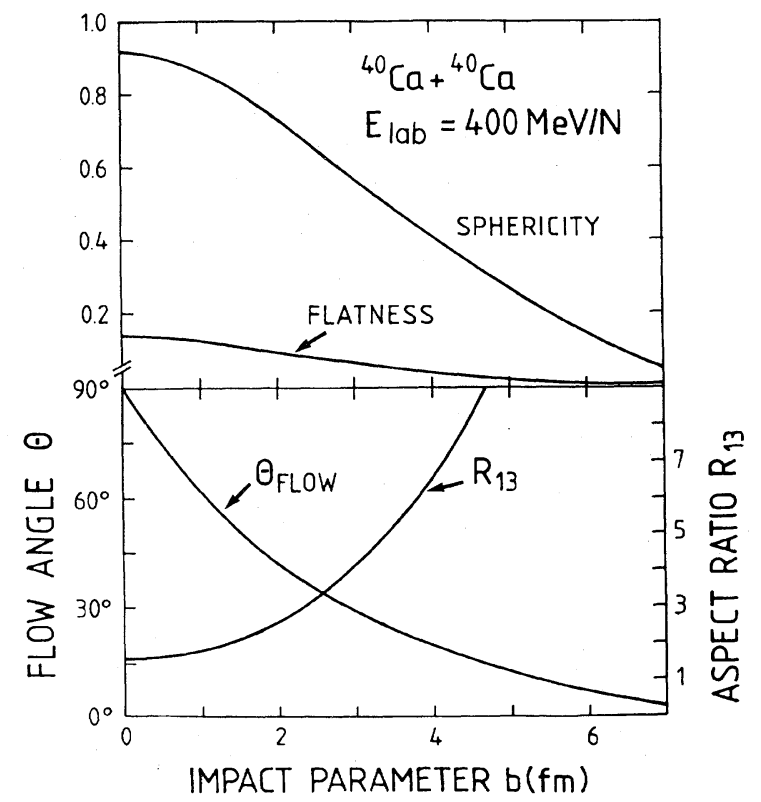

FIG. 2. The impact parameter dependence of $R_{13}, \theta_{\text {flow }}$, sphericity, and coplanarity is shown as calculated for the reaction ${ }^{40} \mathrm{Ca}(400 \mathrm{MeV} /$ nucleon $)+{ }^{40} \mathrm{Ca}$ in the fluid dynamical model.

to the value computed via cascade for this reaction in Ref. 7. However, this coincidence is due only to finite number distortion effects in cascade calculations. As shown in Ref. 10, a sphere sampled randomly by $M$ particles results in $R_{13} \approx 1+3 / \sqrt{M}+22 / M \approx 1.6$ for $M=80$. In hydrodynamics, the limit $M \rightarrow \infty$ is taken. Therefore $R_{13}=1.7$ in hydrodynamics represents true collective flow, while in cascade this value is consistent with an isotropic sphere sampled by 80 particles.

The general behavior of the flow pattern in the fluid dynamical model is as follows: The flow angle rises smoothly from $0^{\circ}$ at large impact parameters to $90^{\circ}$ at $b=0$, while sphericity and coplanarity rise from 0 to 0.9 and 0.2 , respectively. Since the matter flow reflects the longitudinal, $p_{\|}$, and transverse, $p_{\perp}$, momentum transfer in a collision, it can be used to directly measure the pressure built up in the high density stage of the reaction ${ }^{11}$

$$
p_{\perp}=\int_{t} \int_{f} P(\rho, S) d f d t
$$

where $d f$ represents a surface element between the participant and the spectator matters and the total pressure $P(\rho, S)$ is the sum of an interaction pressure $P_{c}(\rho, S=0)$ and a kinetic term $P_{T}(\rho, S>0)$ :

$$
P(\rho, S)=P_{c}(\rho, S=0)+P_{T}(\rho, S>0) .
$$

The bombarding energy dependence of $\left(P_{c}+P_{T}\right) / P_{T}$, i.e., the ratio of the total pressure to the Fermi-gas term, has been calculated in Ref. 11. The results show that there is a strong bombarding energy dependence of $P / P_{T}\left(E_{\text {lab }}\right)$. The kinetic term $P_{T}$ dominates at high energies, $E_{\mathrm{lab}}>1 \mathrm{MeV} /$ nucleon, while the interaction term $P_{c}$ far exceeds $P_{T}$ at intermediate energies, $\$ 200$ 


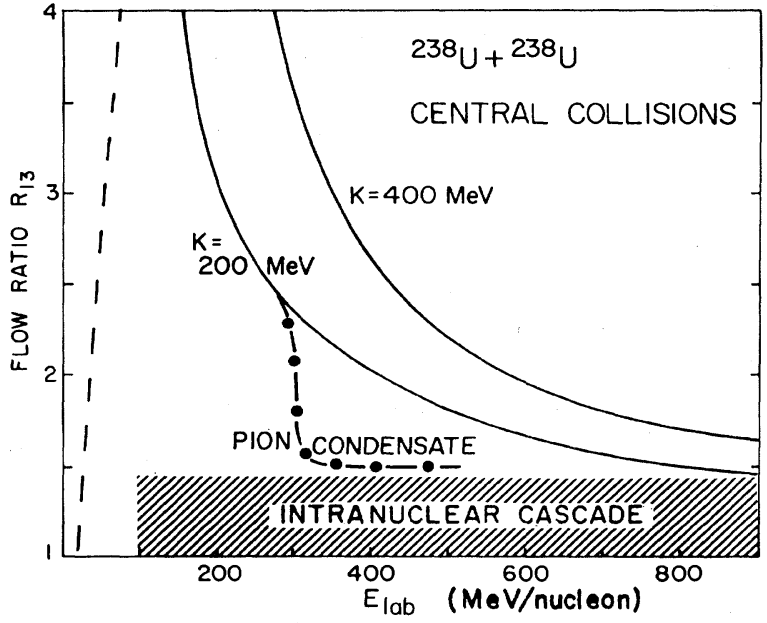

FIG. 3. The bombarding energy dependence of the aspect ratio $R_{13}$ is shown for central collisions of uranium on uranium. The dashed area indicates the results of the cascade calculation; the solid lines represent the results of the fluid dynamical model for different equations of state.

$\mathrm{MeV} /$ nucleon. Second, the total pressure is most sensitive to the stiffness of the nuclear equation of state at energies $\lesssim 200 \mathrm{MeV} /$ nucleon. Since the flow characteristics depend directly on the pressure, we can expect a dependence of the flow pattern on the stiffness of the equation of state. To check this idea we have investigated the bombarding energy dependence of the kinetic flow ratio $R_{13}$ for central collisions of $U+U$ using both the fluid dynamical and the cascade models.

Figure 3 shows the main result of this paper. We find a strong energy dependence of $R_{13}\left(E_{\text {lab }}\right)$ in the fluid dynamical calculation, which indeed closely reflects the energy dependence of $P / P_{T}\left(E_{1 \mathrm{ab}}\right)$ discussed above. This is in stark contrast to the cascade results (shaded area in Fig. 3) that show no appreciable indications for a dependence of $R_{13}$ on the bombarding energy, even for the heavy system $\mathrm{U}+\mathrm{U}$. The values $R_{13}^{\text {cascade }} \lesssim 1.5$ reflect only a globally thermalized "fireball" momentum distribution. In particular, finite number effects ${ }^{10}$ map $R_{13}=1.0$ into $R_{13} \sim 1.2$ for $M=476$. The strong collective flow, as observed in the hydrodynamical calculations, is not seen. At high energies, $E_{\text {lab }}>1 \mathrm{GeV}$, both approaches yield similar values $R_{13}<1.5$. In hydrodynamics $R_{13}$ approaches unity because the random thermal flow $\delta_{i j} E_{T} / 3$ dominates the collective flow $\bar{F}_{i j}$ in Eq. (2) at high energies.

The dependence of $R_{13}\left(E_{\text {lab }}\right)$ on the nuclear compressibility is of particular interest. Figure 3 shows $R_{13}\left(E_{\mathrm{lab}}\right)$ for three different equations of state. As with the dependence of $P / P_{T}$ on the compressibility, $R_{13}$ increases (at a given bombarding energy) if the compressibility [and hence $\left.P_{c}(\rho)\right]$ is increased. This finding shows that global event analysis as a function of beam energy can indeed provide information on the stiffness of the equation of state. The measurement of $R_{13}\left(E_{\text {lab }}\right)$ can also allow for an experimental search for abnormal superdense states (pion condensates, density isomers, in general bends and secon- dary minima in the interaction pressure), which would reveal themselves by a threshold decrease of $R_{13}$ at the critical bombarding energy $E_{\text {lab }}^{\text {crit }}$ sufficient for a transition into an abnormal state to occur. (The decrease of the interaction pressure $P_{c}$ may even lead to metastable density isomeric states. Just above the barrier to such a hypothetical abnormal state, $P_{c}$ would be negative and inhibit an immediate decay of this state. ${ }^{12}$ ) The consideration of such abnormal states is speculative. However, our point is that $R_{13}\left(E_{\text {lab }}\right)$ is a sensitive probe to exotic phenomena as well.

To compare with actual experiments, the ideal hydrodynamic model predictions will have to be corrected for the contribution to $F$ due to nucleons that suffer too few collisions to evolve hydrodynamically. First, there are spectator nucleons that do not suffer any large momentum transfer collisions in the first place. Second, there are knockout nucleons which suffer only on NN collision. Finally, there are intermediate collision nucleons that suffer $2-3$ collisions. These nucleons will be distributed approximately isotropically in the c.m. frame. ${ }^{13}$

To incorporate such nonhydrodynamical background, we decompose $F$ according to the number of collisions made by nucleons

$$
F=\sum_{n=0}^{\infty} P(n) F(n),
$$

where $P(n)$, with $\sum P(n)=1$, is the relative weight of the contribution of nucleons, which collided $n$ times, to $F$, and $F(n)$ is the flow tensor associated with the final distribution of such $n$ collision nucleons in momentum space. We normalize $F$ to unit trace in Eq. (5) by requiring $\operatorname{Tr} F(n)=1$ for all $n$. At best, the flow tensor calculated in hydrodynamics can approximate $F(n)$ for $n \gg 1$. To gain insight into the effect of small $n$ contributions, we divide Eq. (5) into three main terms,

$$
F=p_{0} F_{0}+p_{1} F_{1}+\left(1-p_{0}-p_{1}\right) \bar{F},
$$

where $\bar{F}$ is given by Eq. (2), $p_{0}=P(0)+P(1)$ is the weight of spectator and direct knockout nucleons, and $p_{1}$ is the fraction of nucleons suffering an intermediate number of collisions with $N_{c} \sim 2-3$. The spectator plus knockout contribution $F_{0}$ is approximated by

$$
F_{0}=\operatorname{diag}(0,0,1) \text {, }
$$

since the spectator nucleons and-due to the forwardbackward peaking of the N-N cross section-also the knockout nucleons are concentrated around \pm the incident c.m. momentum per nucleon. ${ }^{13}$ For simplicity we approximate the intermediate collision contribution, $N_{c}=2-3$, by an isotropic momentum distribution for which

$$
F_{1}=\operatorname{diag}\left(\frac{1}{3}, \frac{1}{3}, \frac{1}{3}\right) \text {. }
$$

Finally, for central collisions, for which $\bar{F}$ in Eq. (2) is diagonal,

$$
\bar{F}=\frac{1}{2+r_{h}} \operatorname{diag}\left(1,1, r_{h}\right)
$$


the full tensor has the diagonal form

$$
F=\frac{1}{2+r} \operatorname{diag}(1,1, r) \text {. }
$$

Note, for example, from Fig. 3 that $r_{h} \sim 0.5$ for the $\mathbf{U}+\mathbf{U}$ collision at $400 \mathrm{MeV} /$ nucleon. For $0<r<1, F$ in Eq. (10) describes a pancake shape with aspect ratio $R_{13}=1 / r$ and flow angle $\theta_{F}=90^{\circ}$. For $r>1, F$ describes a cigar shape with $R_{13}=r$ and $\theta_{F}=0^{\circ}$.

The effect of adding $F_{0}$ and $F_{1}$ to $F$ in Eq. (6) is to replace the hydrodynamic value, $r_{h}$, by $r$ given by

$$
\frac{r}{r+2}=p_{0}+p_{1}+\left(1-p_{0}-p_{1}\right) \frac{r_{h}}{2+r_{h}} \text {. }
$$

For example, for $r_{h}=\frac{1}{2}$, i.e., $R_{13}=2$, the measured $r$ exceeds 1 if the fraction of spectator nucleons $p_{0}$ exceeds $\frac{1}{6}$. With $p_{1}=\frac{1}{4}, r>1$ when $p_{0}>\frac{1}{8}$.

Equation (11) shows that the magnitude of $R_{13}$ in Fig. 3 can be significantly reduced as a result of the nonhydrodynamic background contributions. Only a $5 \%$ spectator contribution is required to lower $R_{13}$ from 4 at 300 $\mathrm{MeV} /$ nucleon in Fig. 3 to 2.7. Alternately, a 25\% intermediate isotropic background is sufficient to reduce $R_{13}$ to 2.5 from 4 . Therefore, in comparing data to the hydrodynamic predictions in Fig. 3, the substantial modification of $R_{13}$ due to the background must be taken into account. The presence of the nucleons with $N_{c} \leq 3$ also means that the hydrodynamic calculation should only be started after the few collision initial stage with densities depleted to the value $\rho_{0}\left(1-p_{0}-p_{1}\right)$ instead of using all the nucleons. However, due to the scaling of the hydrodynamic equations with the nucleon number $A$ (i.e., since the results of the calculations are practically independent of $A$ ), we do not anticipate a qualitative change of these results.

A simple way to eliminate the spectator and knockout contributions is to remove nucleons with momenta in a shell of radius $p_{\text {c.m. }}$ and thickness $\Delta \sim 100 \mathrm{MeV} / c$ from the sum of Eq. (1). However, it is not so simple to remove the approximate isotropic background $F_{1}$. One possibility is to estimate that component via an intranuclear cascade calculation. However, a powerful way to get a handle on the background is to measure the double and triple differential distribution for very high multiplicity events directly. ${ }^{2}$ Recall that global event analysis is required for noncentral collisions only because we do not know the reaction plane ahead of time. Only global analysis can provide an estimate of that reaction plane. Once such an estimate is made, however, there is no reason aside from limited statistics not to rotate all events into the same plane and then to display the triple differential cross section, $d^{3} \sigma / d p^{3}$, as suggested in Ref. 2. The triple differential cross section is shown for ${ }^{40} \mathrm{Ca}(400 \mathrm{MeV} /$ nucleon $)+{ }^{40} \mathrm{Ca}$ at $b=2 \mathrm{fm}$ in Fig. 4 . The eigenvalues of $F$ only provide a moment analysis of that triple differential distribution. For central collisions, there is obviously azimuthal symmetry and there is no need to rotate events in the azimuth. In this case $F$ provides a moment analysis effectively only for the double differential distribution $d^{2} \sigma / d E d \cos \theta$. Clearly, $d^{3} \sigma$ contains far more information than $F$. In particular, the spectator contributions and the isotropic

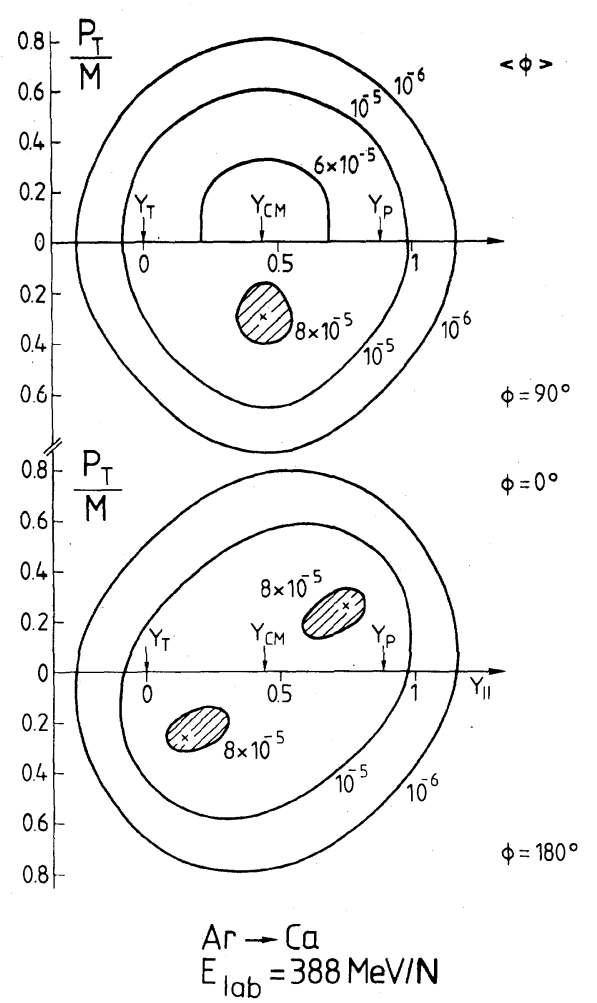

FIG. 4. The triple differential invariant cross section $(1 / E) d^{3} \sigma / d p^{3}$ is shown for ${ }^{40} \mathrm{Ca}(400 \mathrm{MeV} /$ nucleon $)+{ }^{40} \mathrm{Ca}$ at $b=2 \mathrm{fm}$ as obtained from the fluid dynamical calculation.

background may be more easily distinguished from the sidewards enhanced hydrodynamic contribution in that all three components are associated with different phase space regions.

It is interesting to point out that in the first experimental event-by-event analysis, the observed transverse momentum transfer and flow angles considerably exceed the corresponding cascade simulations, even for systems as light as $\mathrm{Ne}+\mathrm{NaF}$ and $\mathrm{Ar}+\mathrm{KCl}$, at energies between 0.4 and $1.8 \mathrm{GeV} /$ nucleon. ${ }^{14}$ The largest deviations from the cascade predictions seem to emerge for the heaviest system studied to date, $\mathrm{Ar}+\mathrm{Pb}$ at 0.4 and 0.8 $\mathrm{GeV} /$ nucleon: The observed average flow angles exceed the maximum flow angle predicted by the cascade by more than $50 \%$.

In conclusion, an event by event analysis of $4 \pi$ exclusive experiments seems to be of great interest in order to explore the flow effects in collisions of heavy nuclei, e.g., $\mathrm{Pb}(200 \mathrm{MeV} /$ nucleon $)+\mathrm{Pb}$. It offers the unique opportunity to study nuclear matter properties at high densities. We have shown that such experiments may be useful to probe the compressibility of dense matter and to search specifically for phase transitions at high densities and temperatures. There are indeed challenging questions to be answered by experiments in the near future.

Note added in proof. Recent data from the plastic ball electronic detection system confirm the theoretically 
predicted strong sidewards peak in the distribution of flow angles in an event-by-event analysis of the reaction ${ }^{93} \mathrm{Nb}$ $(0.4 \mathrm{GeV} /$ nucleon $)+{ }^{93} \mathrm{Nb} .^{15}$
This work was supported by the National Science Foundation, the Department of Energy, and the Bundesministerium für Forschung und Technologie.
${ }^{1}$ R. Stock, H. H. Gutbrod, W. G. Meyer, A. M. Poskanzer, A. Sandoval, J. Gosset, C. H. King, G. King, Ch. Lukner, Nguyen Van Sen, G. D. Westfall, and K. L. Wolf, Phys. Rev. Lett. 44, 1243 (1980).

${ }^{2}$ H. Stöcker, J. A. Maruhn, and W. Greiner, Phys. Rev. Lett. 44, 725 (1980); H. Stöcker, L. P. Csernai, G. Graebner, G. Buchwald, H. Kruse, R. Y. Cusson, J. A. Maruhn, and W. Greiner, Phys. Rev. C 25, 1873 (1982); Phys. Rev. Lett. 47, 1844 (1981).

${ }^{3}$ H. G. Baumgardt, J. U. Schott, Y. Sakamoto, E. Schopper, H. Stöcker, J. Hofmann, W. Scheid, and W. Greiner, Z. Phys. A 273, 359 (1975); J. Hofmann, H. Stöcker, U. Heinz, W. Scheid, and W. Greiner, Phys. Rev. Lett. $\underline{36}, 88$ (1976); H. G. Baumgardt and E. Schopper, J. Phys. Lett. G ㅌ, L231 (1979).

${ }^{4}$ L. P. Csernai, W. Greiner, H. Stöcker, I. Tanihata, S. Nagamiya, and J. Knoll, Phys. Rev. C 25, 2482 (1982).

5 J. I. Kapusta and D. Strottman, Phys. Lett. 103B, 269 (1981).

${ }^{6}$ J. Cugnon, J. Knoll, C. Ridel, and Y. Yariv, Phys. Lett. 109B, 167 (1982).

${ }^{7}$ M. Gyulassy, K. A. Fraenkel, and H. Stöcker, Phys. Lett. 110B, 185 (1982).

${ }^{8}$ H. Stöcker, G. Buchwald, L. P. Csernai, G. Graebner, J. A. Maruhn, and W. Greiner, Nucl. Phys. A387, 205c (1982).

${ }^{9}$ G. Buchwald, G. Graebner, J. Theis, J. Maruhn, W. Greiner, and $\mathrm{H}$. Stöcker, in Proceedings of the International Conference on High Energy Nuclear Physics, Balatonfüred, Hungary, 1983, edited by J. Erö (Central Research Institute for Physics, Budapest) pp. 389-408.
${ }^{10}$ P. Danielewicz and M. Gyulassy, Lawrence Berekeley Laboratory Report LBL-15721, 1983.

${ }^{11} \mathrm{H}$. Stöcker and B. Müller, Z. Naturforsch. (to be published); H. Stöcker, M. Gyulassy, and J. Boguta, Phys. Lett. 103B 269 (1981).

${ }^{12}$ H. Stöcker, J. A. Maruhn, and W. Greiner, Phys. Lett. $\underline{81 B}$, 303 (1979).

${ }^{13}$ Such a decomposition follows from a multiple scattering expansion; see B. Schürmann, M. Hartmann, and H. J. Pirner, Nucl. Phys. A360, 435 (1981).

${ }^{14}$ A. Huie, D. Beavis, S. Y. Fung, W. Gorn, D. Keane, J. J. Lu, R. T. Poe, B. C. Shen, and G. VanDalen, Phys. Rev. C 27, 439 (1983); H. Stroebele, R. Brockmann, J. W. Harris, F. Riess, A. Sandoval, R. Stock, K. L. Wolf, H. G. Pugh, L. S. Schroeder, R. E. Renfordt, K. Tittel, and M. Maier, Phys. Rev. C 27, 1349 (1983); D. Beavis, S. Y. Chu, S. Y. Fung, W. Gorn, A. Huie, D. Keane, J. J. Lu, R. T. Poe, B. C. Shen, and G. VanDalen, ibid. 27, 2443 (1983); H. H. Gutbrod, H. Lohner, A. M. Poskanzer, T. Renner, H. Riedesel, H. G. Ritter, A. Warwick. F. Weik, and H. Weiman, Phys. Lett. 127B, 317 (1983)

${ }^{15}$ H. G. Ritter, H. A. Gustafsson, H. H. Gutbrod, B. Kolb, H. Löhner, B. Ludewigt, A. M. Poskamzer, T. Renner, H. Riedsel, A. Warwick, F. Weik and H. Wieman, in Proceedings of the International Conference on High Nuclear Physics, Balatonfüred, Hungary, 1983, edited by J. Erö (Central Research Institute for Physics, Budapest) pp. 275-279. 